\title{
ZnSe quantum dots through a facile one pot synthesis process
}

\author{
Irtiqa Syed and Santa Chawla*
}

\author{
Luminescent Materials Group, CSIR-National Physical Laboratory, Dr.K.S.Krishnan Road, New \\ Delhi - 110012, India \\ *santa@nplindia.org
}

Keywords: Quantum Dots; ZnSe; Oleic Acid; Photoluminescence; Bandgap

\begin{abstract}
A novel one pot synthesis approach in oleic acid medium was employed to obtain monophasic ZnSe quantum dots (QD) of average size 3.7nm. The QDs were well crystalline in hexagonal phase as revealed by x-ray diffraction and high resolution transmission electron microscopy (HRTEM) studies. The ZnSe QDs exhibit sharp emission peak in the blue (465nm) with 385picosecond fluorescence decay time. The theoretical band gap corresponding to $3.7 \mathrm{~nm}$ ZnSe QDs matched well with the measured $3.11 \mathrm{eV}$ band gap of synthesized QDs which thus showed quantum confinement effect.
\end{abstract}

\section{Introduction}

Semiconductor quantum dots (QDs) exhibit unique optical properties where the emission colour can be tuned by tailoring the particle size as a manifestation of quantum confinement effect. QDs have grown in popularity due to their wide applicability ranging from solid state electronics, in the manufacture of sensors [1-3] to as far as biomedical research as unique fluorescent labels [4-6]. Among the variety of II-VI semiconductor QDs, which are widely synthesized for their tunable emissions, $\mathrm{ZnSe}$ offers potential as blue emitting semiconductor for lasing and bio-marking, due to its bulk band of $2.69 \mathrm{eV}$ and Bohr exciton radius of 5.7nm [7] and also being less toxic among other Cd chalcogenides.

Previous reports in literature involve the synthesis of hydrophobic ZnSe QDs and water soluble ZnSe QDs by an aqueous route. Hydrophobic QDs require organo-metallic precursors such as dimethyl cadmium and diethyl zinc with a chalcogenide anion source. The method requires rapid injection of a solution of chemical reagents into a hot and vigorously stirred organic solvents, that can coordinate with the surface of precipitated QDs, at reaction temperature $250-300^{\circ} \mathrm{C}$. QD's prepared by this method require further functionalization with organic molecules possessing thiol, carboxy or amino group to improve their solubility [8]. The aqueous synthesis route consists in reaction of heavy metal ( $\mathrm{Zn}, \mathrm{Cd}$ etc.) precursor with chalcogen precursor in water phase with very long reaction time which can range from several hours to several days. The QDs synthesised through aqueous route synthesis often have surface defects and dangling bonds which need to be eliminated by using capping agents such as thioglyvolic acid (TGA) [9, 10].

Highly fluorescent ZnSe QDs were synthesized in aqueous solution in presence of thiol compounds, mercaptosuccinic acid with quantum efficiency up to $44 \%$ at $407 \mathrm{~nm}$ under $325 \mathrm{~nm}$ UV excitation [11]. In thin film form, $\mathrm{ZnSe}$ and $\mathrm{Cu}$-doped nanoparticle multilayer composite films have been prepared by Hao et al $[12,13]$. ZnSe quantum dots have also been successfully prepared in silica thin films via sol-gel process [14]. Reiss et al. [15] have reported the synthesis of ZnSe nanoparticles in non-coordinating solvent (octadiene) via direct reaction of zinc stearate with selenium. There has also been direct synthesis of $\mathrm{ZnSe}$ via an aqueous solution route and many of the capping agents used were thiophenol, thiourea, mercapto acetate etc. which are toxic and will pollute the environment if the synthesis involves large scale production [16, 17]. Water-soluble $\mathrm{ZnSe}: \mathrm{Mn} / \mathrm{ZnS}$ core/shell d-dots with pure dopant emission were synthesized via a new two-step process by rationally tailoring the experimental parameters, the highly luminescent and colortunable $\mathrm{ZnSe}: \mathrm{Mn} / \mathrm{ZnS}$ d-dots with core/shell structure were obtained by Bohua et al [18]. 
Chemical routes for the synthesis of ZnSe QDs reported thus far involve the use of expensive reagents and toxic reducing agents such as phosphine. We present a low cost, facile one pot phosphine free synthesis of $\mathrm{ZnSe}$ QDs at moderate temperature. The method allows for an environmental friendly synthesis of $\mathrm{ZnSe}$ QDs without the use of toxic external reducing agents by using oleic acid, a naturally commonly occurring hydrocarbon. The synthesized ZnSe QDs show wider band gap as compared to its bulk counterpart and sharp blue emission.

\section{Experimental}

One pot synthesis of ZnSe quantum dots. Synthesis was carried out using analytical grade Zinc acetate, Oleic acid (Merck) and Selenium (Aldrich). Reagents were used without further purification. In a typical procedure $0.02 \mathrm{M}$ of zinc acetate and selenium powder along with $100 \mathrm{ml}$ of oleic acid were stirred together in a three neck flask. The setup was heated to $110^{\circ} \mathrm{C}$ after being evacuated, followed by nitrogen bubbling. The temperature was raised to $250^{\circ} \mathrm{C}$ after 30 minutes of vigorous stirring and this was maintained for four hours in an inert atmosphere. The mixture was refluxed throughout the heating process. The reaction progress can be ascertained by a change of colour of the solution to yellow. The resulting precipitate was washed with ethanol, acetone and water multiple times by ultrasonic dispersion-centrifugation cycles.

The reaction progresses within the organic solvent with the dissolution of elemental selenium in oleic acid at moderate temperatures. The dissolved selenium then begins to react with the zinc precursor, zinc acetate when the temperature is increased to $250^{\circ} \mathrm{C}$. The distinctive trait of the procedure is the lack of use of an external, environmentally toxic reducing agent that usually follows the synthesis of quantum dots. Oleic acid possessing adequate thermal stability allows for solvation of reagents and provides optimal capping of the formed products from external influence such as oxidation. The final product of QDs obtained is monodisperse and monophasic.

Characterization. Phase characterization of synthesized QDs in powder form were done by X-ray powder diffraction in the $2 \theta$ range of $15-80^{\circ}$ on a Rigaku MiniFlex Diffractometer using $\mathrm{Cu} \mathrm{K}_{\alpha}$ radiation. Grain morphology and size distribution was studied by Transmission Electron Microscopy (TEM) with a FEI TECNAI F 30 TWIN, TECNAI transmission electron microscope, using an accelerating voltage of $300 \mathrm{kV}$. The photoluminescence (PL) excitation, emission spectra and time resolved decay of luminescence were measured using combined steady state fluorescence and lifetime spectrometer of Edinburgh Instruments (model FLSP920) with Xe lamp as excitation source. Luminescence decay measurements were carried out using excitation of picoseconds pulsed UV diode laser and employing time correlated single photon counting (TCSPC) technique. UVVisible absorption measurements of ZnSe colloidal solution were carried out using Avantes UVVisible spectrometer.

\section{Results and Discussion}

Structural Analysis. The XRD pattern of ZnSe quantum dots prepared in oleic acid (Fig. 1) matched well with standard pure hexagonal wurtzite structure (JCPDS 15-0105). The average crystallite size was calculated using Debye-Scherer formula and was found to be $3.7 \mathrm{~nm}$, indicating the formation of quantum dots. 


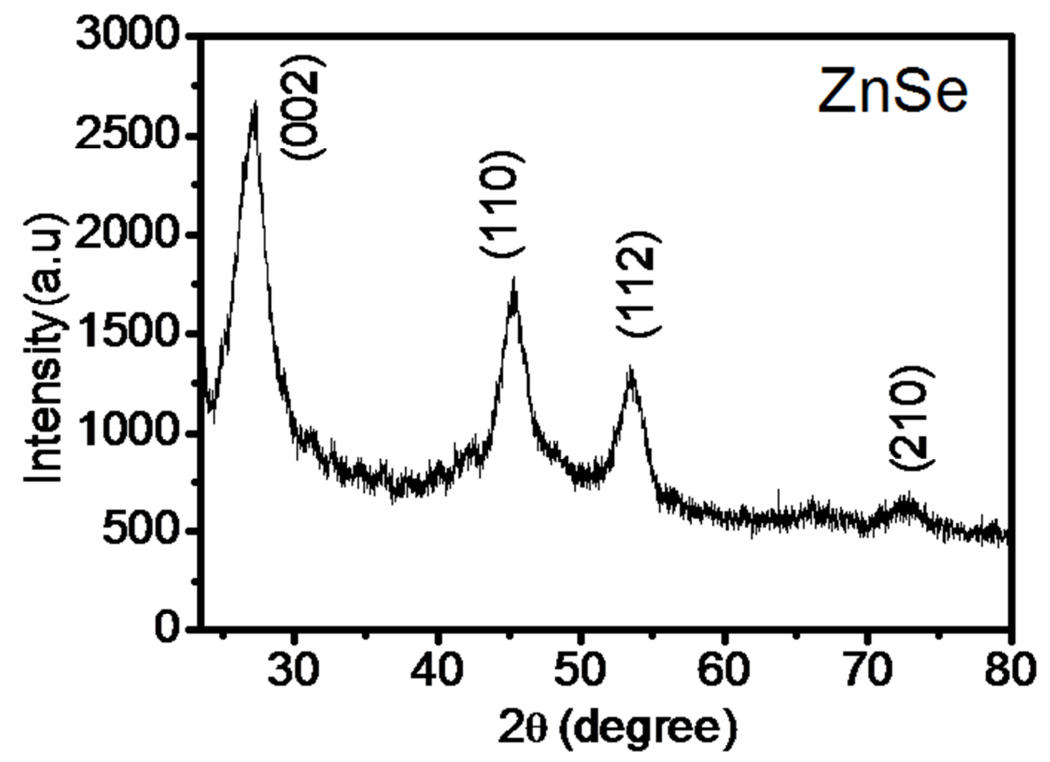

Fig. 1 XRD pattern of ZnSe synthesized in Oleic Acid medium showing well formed broad peaks that could be indexed to hexagonal structure.

Morphology. TEM and HRTEM image of ZnSe synthesized in Oleic Acid medium is shown in Fig. 2 (a) and (b) respectively. The formation of QDs of size in the range of 3-5 nm is apparent and individual QD is well crystalline as revealed by lattice fringes whose separations conform to hexagonal lattice planes of $\mathrm{ZnSe}$.
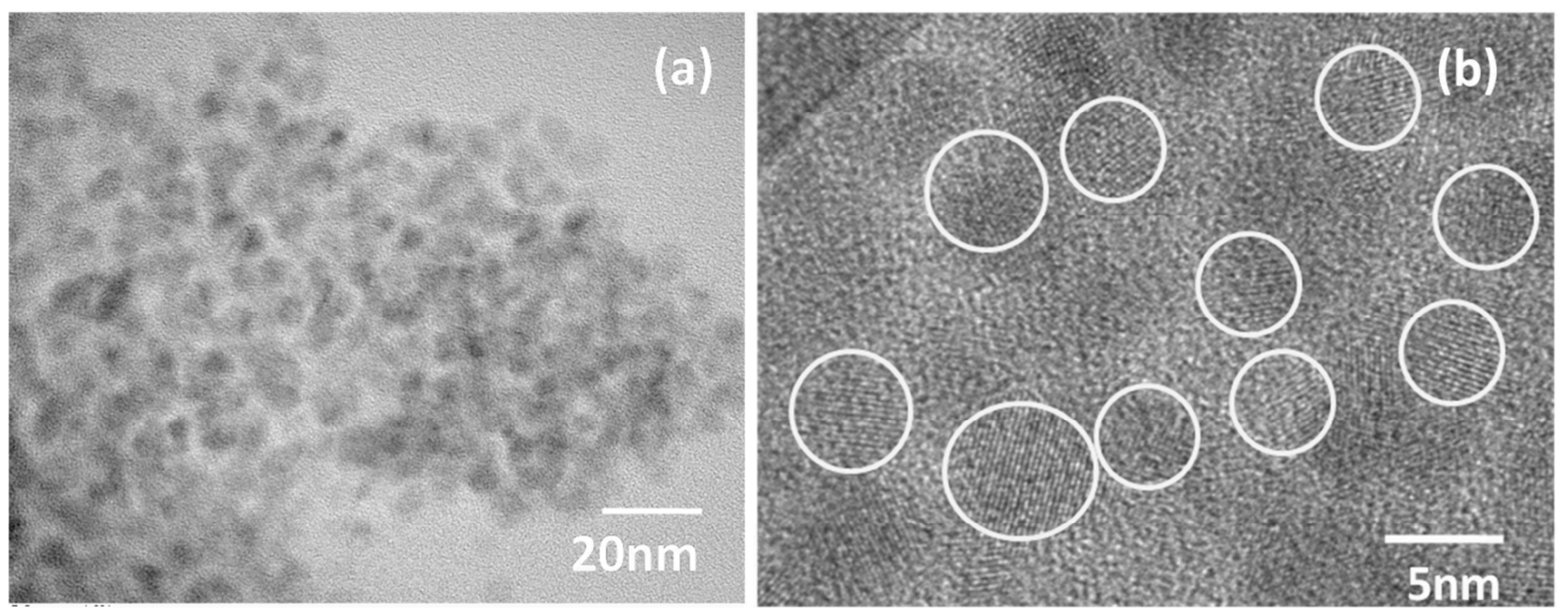

Fig. 2 (a) TEM and (b) HRTEM image of ZnSe synthesized in Oleic Acid medium. HRTEM images show well crystalline quantum dots with distinct lattice fringes.

Photoluminescence. Photoluminescence excitation and emission spectra of synthesized $\mathrm{ZnSe}$ quantum dots colloidal solution is shown in Fig. 3(a). PL emission spectra of synthesized ZnSe quantum dots show a sharp emission peak in the blue $(465 \mathrm{~nm})$ when excited at $309 \mathrm{~nm}$ as shown in Figure 3a. The corresponding PL excitation spectra recorded at $465 \mathrm{~nm}$ emission wavelength exhibit a sharp peak at 309nm (Fig.3a). Inset in Fig. 3(a) shows the digital photograph of ZnSe QD colloid in a quartz cuvette under $309 \mathrm{~nm}$ UV light, clearly displaying the blue emission from the QDs. At room temperature, the excitation peak of $\mathrm{ZnSe}$ quantum dots is blue shifted compared to bulk $\mathrm{ZnSe}$. The shifting of peaks to higher energies is a manifestation of quantum confinement effect as quantum dots have average size around $3.7 \mathrm{~nm}$ and Bohr exciton radius of $\mathrm{ZnSe}$ is $5.7 \mathrm{~nm}$. Blue PL emission at deep UV excitation $(309 \mathrm{~nm})$ corresponds to higher excited states. The linewidth of 
excitation and emission peaks of ZnSe QDs are very narrow with full width at half maxima about $10 \mathrm{~nm}$ which suggest very narrow size distribution of the synthesized QDs. Such narrow peaks and UV blue emission has also been reported from cubic ZnSe QDs with size in the range 3.2 to $4.5 \mathrm{~nm}$ synthesized via hydrothermal route [19].

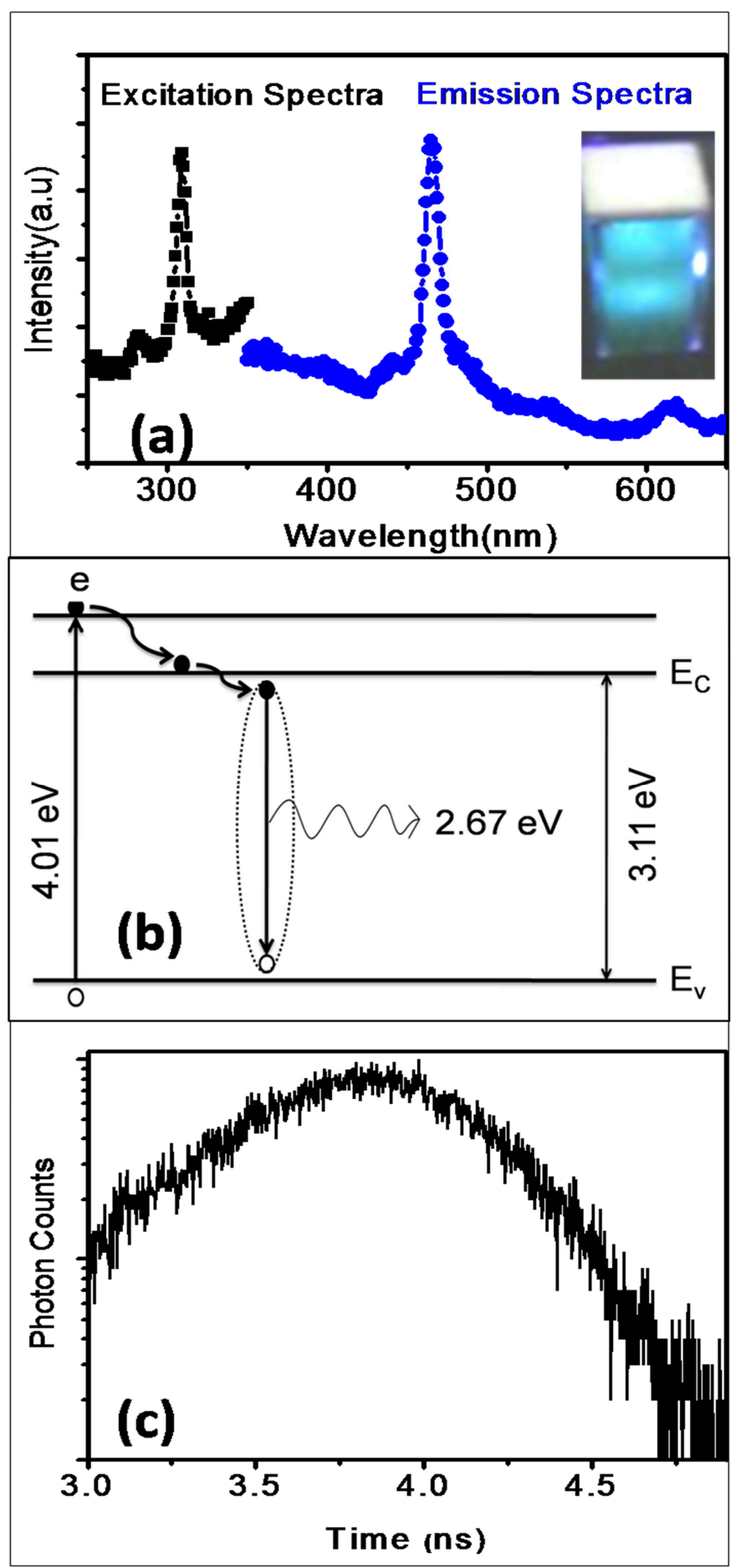

Fig.3 (a) PL excitation spectra taken at 465nm emission peak and emission spectra when ZnSe colloid is excited by 309nm UV light corresponding to excitation peak; (b) energy level diagram indicating the excitonic recombination leading to sharp blue emission;(c)TRPL of ZnSe QDs at

$$
465 \mathrm{~nm} \text {. }
$$

The band diagram showing excitation and emission processes are shown in Fig. 3(b). Absorption of energy by the ZnSe QDs at $4.01 \mathrm{eV}$ clearly suggests excitation at a level higher than the band gap due to quantum confinement effect. Since the sizes of quantum dots are smaller than the Bohr radius, quantum confinement is achieved which also influences the excitation at higher 
energy. The sharp emission peak at $465 \mathrm{~nm}(2.67 \mathrm{eV})$ can be attributed to band edge excitonic emission of $\mathrm{ZnSe}$. The emission peak is Stokes shifted to a higher wavelength due to loss in vibrational energy when electrons relax to bottom of conduction band or form an exciton close to band edges that radiatively recombine to emit fluorescence as shown in Fig. 3(b).

Time Resolved Photoluminescence (TRPL). The time between excitation and recombination is known as the optical recombination time or photoluminescence lifetime. The decay time was measured by time correlated single photon counting technique by repetitively exciting the colloidal sample using a picoseconds pulsed UV laser. Scanning over many pulses, the resultant decay curve viewed on a semilogarithmic scale, indicate the monoexponential decay as shown in Fig. 3 (c). As the exciting pulse was extremely short $(\sim 200 \mathrm{ps})$, time resolved measurement showed a finite rising edge due to the exciting pulse. The decay curve has been fitted into mono exponential equation with decay time (1/e decay time i.e., time taken to decay to $37 \%$ of the peak value) 384.8 picosecond. The decay time reflects the average time span that the excited electron spends before recombining with a hole thus emitting a photon. Such short decay time indicates that the photophysical process likely involves radiative recombination of nearly free electron hole pairs as well as nonradiative charge transfer.

UV Visible Spectroscopy. UV Visible transmission spectra of ZnSe quantum dots dispersed in oleic acid have been recorded and are shown in Fig. 4, corresponding Tauc plot is shown in the inset. The Tauc plot of a direct band gap semiconductor $\mathrm{ZnSe}$ is plotted from the measured absorption coefficient as shown; the absorption coefficient is obtained by dividing the measured absorbance by the path length i.e. thickness of cuvette. The best linear fit is plotted for the Tauc plot. The band gap is obtained by extrapolating the linear portion of the Tauc plot to be $3.11 \mathrm{eV}$. The absorption edge of ZnSe QDs is blue shifted to higher energy compared to that of bulk ZnSe due to quantum confinement effect. The estimated band gap value from Tauc plot $(3.11 \mathrm{eV})$ is also blue shifted from the bulk band gap of $\mathrm{ZnSe}(2.69 \mathrm{eV})$.

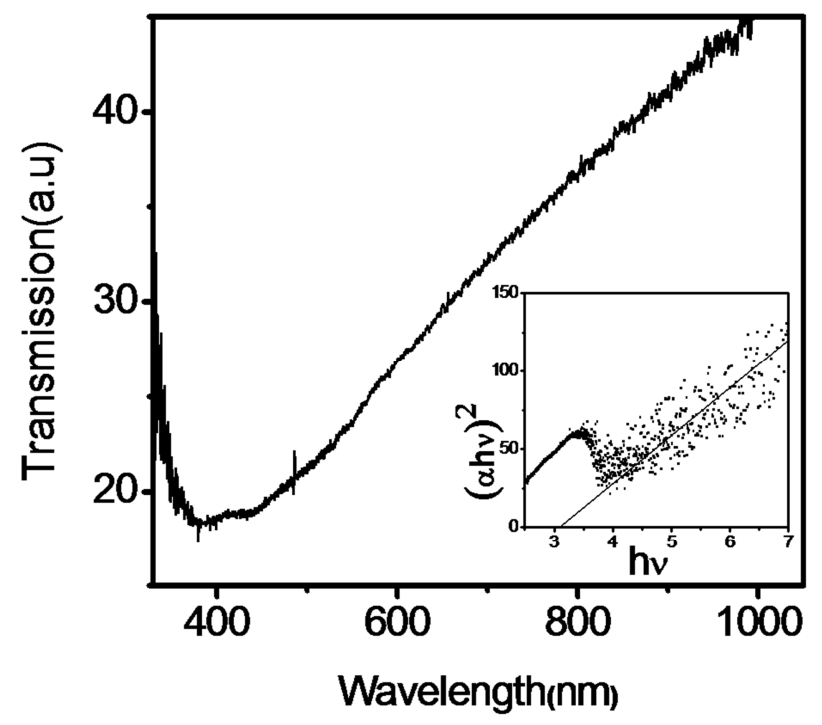

Fig. 4 UV-visible transmission spectra of ZnSe quantum dot colloid, inset shows Tauc Plot for estimation of band gap

Theoretical band gap calculation. Size dependent band gap gives an opportunity to estimate the nanocrystal size from UV-visible absorption spectrum by using the Brus equation for change in the band gap energy with the change in particle size:

$$
E_{g}=E_{\text {gbulk }}+h^{2} / 4 r^{2}\left(1 / m_{e}^{*}\right)
$$


Where $\mathrm{E}_{\text {gbulk }}$ for $\mathrm{ZnSe}$ is $2.69 \mathrm{eV}$, the size of quantum dots was estimated from XRD to be $3.7 \mathrm{~nm}$. Putting these values in the Brus equation gave us a theoretical value of bandgap equal to $3.1 \mathrm{eV}$, which is similar to that of the experimentally estimated band gap. The band gap of bulk $\mathrm{ZnSe}$ is $2.69 \mathrm{eV}$ but with decrease in particle size it has increased to $3.11 \mathrm{eV}$ which clearly shows the quantum confinement effect for synthesized $3.7 \mathrm{~nm} \mathrm{ZnSe}$ quantum dots.

\section{Summary}

A novel one pot synthesis method using oleic acid as reaction medium yielded hexagonal monophasic ZnSe quantum dots of size $3.7 \mathrm{~nm}$. The $\mathrm{ZnSe}$ QDs show quantum confinement effect and sharp blue emission that decays in hundreds of picoseconds. Such novel and cost effective technique can be useful for preparing large quantities of ZnSe QDs for various applications such as light emitting diodes, fluorescent biological tags, photodetectors and full color display. ZnSe Quantum Dots have a large binding energy making them ideal choice as inorganic passivating shell for various semiconductor core nanocrystals, in order to improve their emission properties and to enhance their stability.

\section{References:}

[1] L. J. Zhang, C. L. Xu and B. X. Li, Microchim. Acta; 2009, 166, 61-68.

[2] T. Li, Y. Y. Zhou, J. Y. Sun, D. B. Tang, S. X. Guo and X. P. Ding; Microchim. Acta, 2011, 175, 113-119.

[3] L. Wang, R. Yang, J. Li, L. Qu; Anal. Methods, 2014, 6, 3449-3455.

[4] Y. L. Li, J. Zhou, C. L. Liu and H. B. Li; J. Mater. Chem., 2012, 22, 2507-2511.

[5] W. R. Algar, A. J. Tavares and U. J. Krull; Anal. Chim. Acta, 2010, 673, 1-25.

[6] J. A. Chen, Y. Pei, Z. W. Chen and J. Y. Cai; Micron, 2010, 41, 198-202.

[7] F. T. Quinlan, W. Tremel, S. Risbud, P. Stroeve; Langmuir, 2000, 16, 8.

[8] J. Tian, R. Liu, Y. B. Zhao, Q. Xu and S. Zhao; J. Colloid Interface Sci., 2009, 336, 504-509.

[9] Y. He, H. T. Lu, L. M. Sai, W. Y. Lai, Q. L. Fan, L. H. Wang, W. Huang; J. Phys. Chem. B, 2006, 110, 13370-13374.

[10] H. Zhang, Z. Zhou, B. Yang and M. Y. Gao; J. Phys. Chem. B, 2003, 107, 8-13.

[11] G. Y Lan, Y. W Lin, Y. F Huang, H. T. Chang; J. Mater. Chem, 2007, 17, 2661-2666.

[12]E. Hao, H. Zhang, B. Yang, H. Ren, and J. Shen; J. Colloids Interf. Sci., 2001, 238, 285-290.

[13]H. Jiang, X. Yao, J. Che, M. Wang, F. Kong; Ceram. International, 2004, 30, 1685-1689.

[14] Y. Wang, X. Yao, M. Wang, F. Kong, and J. He; J. Cryst. Growth, 2004, 268, 580-584.

[15]P. Reiss, G. Quemard, S. Carayon, J. Bleuse, F. Chandezon, A. Pron; Mater. Chem. Phys., 2004, 84, 10.

[16]J. H. Li , C. L. Ren, X. Y. Liu , Z. D. Hu , D. S. Xue; Mater Sci Eng A, 2001, 458,319.

[17]Q. L Wei , S. Z Kang, Mu J.; Colloid Surf A, 2004, 247,125.

[18] D. Bohua , C. Lixin, S. Ge, L. Wei; J. Phys. Chem. C, 2012, 116, 12258-12264.

[19]L. L. Peng, Y.H. Wang, C.Y. Li, J. Nanosci. Nanotech.2010, 10(3):2113-8. 